\title{
Hematoma intramuscular espontâneo no dorso de paciente com doença hepática crônica - relato de caso
}

\section{Spontaneous intramuscular hematoma on back of patient with chronic hepatic disease - case report}

Maria Laryssa da Silva Pontes ${ }^{1}$, Evelliny Gomes da Silva ${ }^{2}$, Bruno Vinicius Nascimento da Silva ${ }^{3}$, Phablo Fernandes José Oliveira Ferreira ${ }^{3}$.

${ }^{1}$ Residente de Clínica Médica do Hospital Getulio Vargas, Recife, Pernambuco, Brasil

${ }^{2}$ Universidade Federal de Pernambuco, Campos Acadêmico do Agreste, Recife, Brasil

${ }^{3}$ Universidade Federal de Pernambuco, Recife. Brasil

\begin{abstract}
Resumo
A cirrose hepática é uma das principais causas de sangramento espontâneo dado o desequilíbrio da hemostasia e hemodinâmica dos portadores da doença. Hematomas intramusculares espontâneos principalmente na região do dorso não são uma complicação comum da cirrose, portanto, apresentam prognóstico reservado. Além disso, a presença de hemotórax em pacientes cirróticos é também justificada pela coagulopatia da doença, sendo mais rara que o hematoma intramuscular. Neste relato será abordado o caso de um paciente com quadro de dor lombar e escapular direita que evoluiu com astenia, hiporexia e icterícia. Foi internado, colhido exames laboratoriais e feitos exames complementares, sendo diagnosticado com hematoma intramuscular espontâneo em dorso e hemotórax espontâneo, optou-se pela conduta conservadora. Os registros de hematomas intramusculares e hemotórax espontâneos são raros e, por esse motivo, o manejo de cada caso é individualizado. Com isso, é importante considerar o hematoma intramuscular como uma complicação grave associada a Cirrose Hepática Alcoólica.

Palavras-chave: Hematoma, Cirrose Hepática Alcoólica, Hemotórax, Tratamento Conservador
\end{abstract}

\begin{abstract}
Liver cirrhosis is one of the main causes of spontaneous bleeding due to hemostasis and hemodynamics imbalance in patients with the disease. Spontaneous intramuscular hematomas, especially in the dorsal region, are not a common complication of cirrhosis, therefore, they have a poor prognosis. In addition, the presence of hemothorax in cirrhotic patients is also justified by the coagulopathy of the disease, being more rare than intramuscular hematoma. This report will approach the case of a patient with low back and right scapular pain who developed asthenia, hyporexia and jaundice. He was admitted, laboratory and complementary tests were performed, being diagnosed with spontaneous intramuscular hematoma on the back and spontaneous hemothorax, a conservative approach was chosen. The records of spontaneous intramuscular hematomas and hemothorax are rare and, for this reason, the management of each case is individualized. Therefore, it is important to consider intramuscular hematoma as a serious complication associated with alcoholic liver cirrhosis.
\end{abstract}

Keywords: Hematoma, Alcoholic Liver Cirrhosis, Hemothorax, Conservative treatment.

\section{Introdução}

A cirrose hepática é considerada uma das principais causas de sangramento espontâneo, justificado por desequilíbrios da hemostasia e hemodinâmica, que podem culminar em sangramentos ou trombose. Algumas alterações 
encontradas nesses indivíduos são: hipertensão portal, trombocitopenia, hipoprotrombinemia e queda na produção de fatores coagulatórios ${ }^{1}$. As perdas sanguíneas costumam acontecer no trato gastrointestinal, nariz e boca e são mais associadas com doença mais avançada, com função hepática comprometida $^{1}$. A formação de hematomas intramusculares pode ocorrer de forma traumática ou em pacientes com hemofilia ou prosseguimento de terapia anticoagulante ${ }^{1}$. Quando secundários à hepatopatia crônica, geralmente são associados à etiologia alcoólica ${ }^{1}$ e possibilidade de evolução em choque hipovolêmico ${ }^{2}$. Hematomas intramusculares como consequência da cirrose hepática são pouco comuns ${ }^{3}$, os localizados na região póstero-lateral direita como o deste caso são ainda mais raros havendo poucos relatos disponíveis. A mortalidade associada à essa complicação diverge na literatura, com fontes afirmando que o tempo de diagnóstico e grau de cirrose não estão diretamente relacionados com a letalidade ${ }^{1}$. Já a definição de hemotórax é a presença de líquido pleural com hematócrito maior que $50 \%$ do hematócrito do sangue periférico e a maioria é causado por trauma, após procedimentos cirúrgicos ou por coagulopatias/uso de anticoagulação, sua ocorrência espontânea é bastante rara ${ }^{4}$. Mesmo entre cirróticos com história de sangramentos a ocorrência de hemotórax espontâneo é mais raro ainda que os hematomas intramusculares e possui mortalidade desconhecida dado a escassez de relatos. Logo, tendo vista que a ocorrência de hemotórax e hematoma intramuscular espontâneos (HIE) juntos em um paciente com situação hemodinâmica alterada e de manejo difícil é um evento raro, que essas complicações são pouco abordadas na literatura e não possuir um consenso sobre a melhor abordagem terapêutica ${ }^{3}$ e podem possuir alta mortalidade, está sendo apresentado este relato de caso, com o objetivo de trazer à luz do debate e criar maior consciência entre a comunidade médica.

\section{Relato de caso}

Paciente, 38 anos, sexo masculino iniciou quadro de dor em forte intensidade em região lombar e ombro direito, associado a febre não aferida. Referiu que, 2 dias após o início dos sintomas, evoluiu com astenia, hiporexia e icterícia importante em esclera e mucosas, decidindo procurar a emergência na sua cidade de origem. Foi encaminhado para um hospital terciário para melhor avaliação do quadro. Foi admitido e realizou-se exames laboratoriais, representados em Tabela 1, que evidenciaram anemia macrocítica grave, plaquetopenia e hiperbilirrubinemia e assim realizou-se hemotransfusão (2 concentrados de hemácias + plasma fresco).

Tabela 1: Exames laboratoriais realizados na Admissão.

\begin{tabular}{ll}
\hline Exame & Resultado \\
\hline $\mathrm{Hb}$ & $3,7 \mathrm{~g} / \mathrm{dL}$ \\
$\mathrm{Ht}$ & $11,1 \%$ \\
$\mathrm{VCM}$ & $115,6 \mathrm{fL}$ \\
$\mathrm{HCM}$ & $38,5 \mathrm{pg}$ \\
$\mathrm{RDW}$ & $14 \%$
\end{tabular}




$\begin{array}{ll}\text { Plaquetas } & 71.000 / \mathrm{mm}^{3} \\ \text { Vitamina B12 } & 1.962 \mathrm{ng} / \mathrm{mL} \\ \text { TP } & 28,7 \\ \text { INR } & 2,74 \\ \text { BT } & 11,64 \mathrm{mg} / \mathrm{dL} \\ \text { BD } & 7,07 \mathrm{mg} / \mathrm{dL} \\ \text { BI } & 4,57 \mathrm{mg} / \mathrm{dL}\end{array}$

Foi então transferido à enfermaria de Clínica Médica para dar seguimento à investigação. Para descobrir a etiologia do quadro foi realizado ultrassonografia (USG) de abdome superior com doppler colorido de fígado e sistema porta, que evidenciou a presença de hepatopatia crônica, ascite (discreta) e derrame pleural direito. Em seguida, foi realizado endoscopia digestiva alta (EDA) que não encontrou focos de sangramentos ativos e calculouse os escores de função hepática, definindo Child C/MELD 25. Ademais, diante de clínica compatível com hepatite alcóolica, calculado Maddrey 69, porém foi optado por não iniciar corticoterapia, visto histórico duvidoso de sangramento recente. Seguiu em observação para vigilância de sinais de encefalopatia, sangramentos, infecções, piora da função renal (risco de síndrome hepatorrenal) e distúrbios hidroeletrolíticos. Nesse ínterim, evoluiu no internamento com dor em escápula direita, ombro direito, hemitórax direito e presença de desnível em região escápula-dorso, sem presença de trauma. Com isso, foi realizado tomografia computadorizada (TC) de tórax (Figura 1) que sugeriu hematoma/hemotórax, sendo usada em seguida uma USG no local, que evidenciou coleção sólido-cística, de 16,4 x 13,0 cm na parede pósterolateral direita em plano muscular profundo compatível com hematoma intramuscular. Após discussão do caso com cirurgia torácica e radiologia, foi optado por conduta conservadora, sob risco do sangramento se perpetuar após descompressão de hematoma. Sendo assim, foi mantido apenas com controle álgico.

Figura 1. Tomografia de tórax do dia 18/07/2019

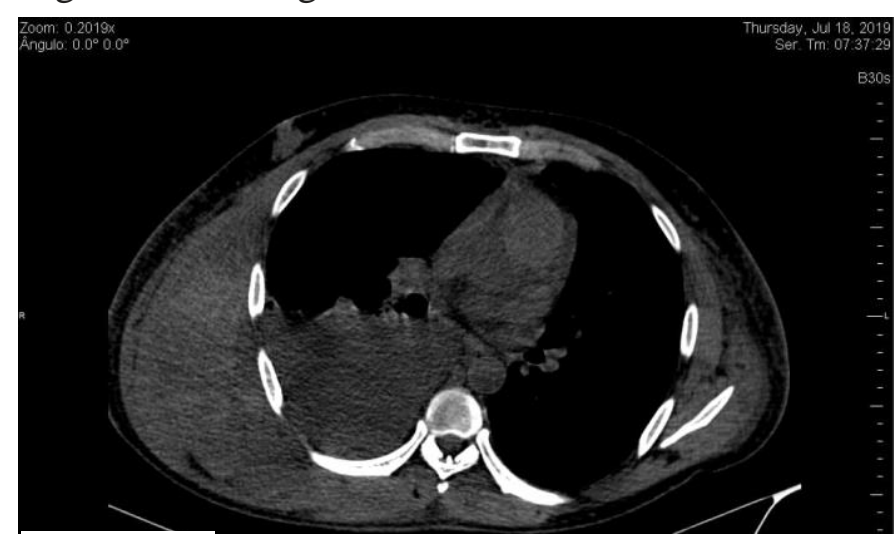

(admissão).

Como a $\mathrm{Hb}$ estava em queda $(6.8 \mathrm{~g} / \mathrm{dL})$, porém assintomático, paciente foi transfundido, mantendose posteriormente sem novos episódios de queda de níveis hematimétricos. Ademais, paciente evoluiu com melhora clínica e laboratorial (Tabela 2) da hepatopatia alcoólica e, com redução tanto do HIE quanto do hemotórax (Figura 2) sendo optado por alta hospitalar após um mês de internamento e se encontra, atualmente, em acompanhamento ambulatorial após um ano do internamento. 


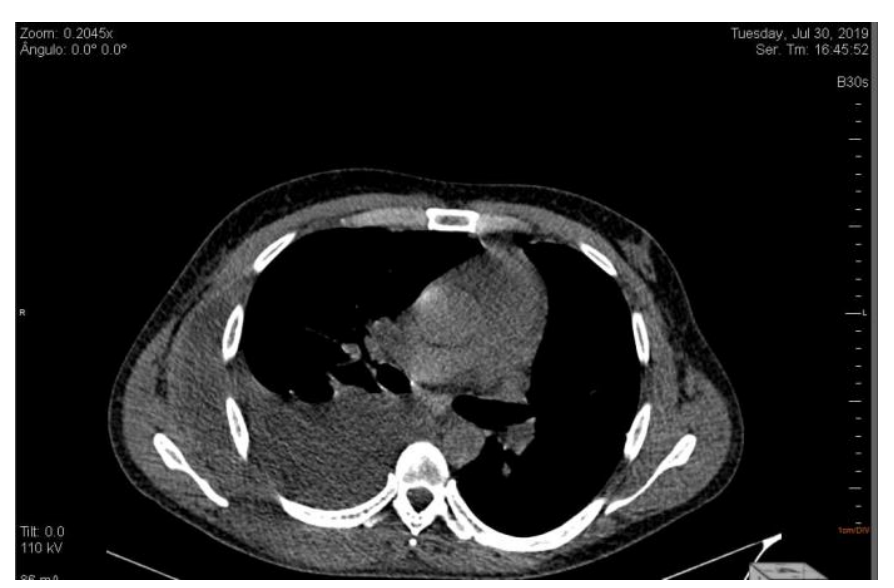

Figura 2: Tomografia de tórax do dia 30/07/2019 indicando regressão tanto do HIE quanto do hemotórax.

Tabela 2: Exames laboratoriais da alta.

\begin{tabular}{ll}
\hline Exame & Resultado \\
\hline $\mathrm{Hb}$ & $8,4 \mathrm{~g} / \mathrm{dL}$ \\
$\mathrm{Ht}$ & $23,7 \%$ \\
$\mathrm{VCM}$ & $99,2 \mathrm{fL}$ \\
$\mathrm{HCM}$ & $35,1 \mathrm{pg}$ \\
RDW & $17,9 \%$ \\
Plaquetas & $88.000 / \mathrm{mm}^{3}$ \\
TP & $23 \mathrm{~s}$ \\
INR & 2,01 \\
BT & $7,50 \mathrm{mg} / \mathrm{dL}$ \\
BD & $3,90 \mathrm{mg} / \mathrm{dL}$ \\
BI & $3,60 \mathrm{mg} / \mathrm{dL}$ \\
\hline
\end{tabular}

\section{Discussão}

$\mathrm{Na}$ literatura encontra-se ao pesquisar sobre o tema relatos de caso avulsos e algumas séries de casos, mas nunca grandes revisões ou guidelines que ajudem aos médicos como lidar adequadamente com essas consequências hemorrágicas em pacientes cirróticos, pois são mais comuns em usuários de medicamentos anticoagulantes ou que possuam hemofilia ${ }^{1}$. Durante a busca em bases de dados encontramos que os HIE podem acontecer em vários locais, mas houve certa preponderância nos músculos iliopsoas e reto abdominal como pode ser visualizada na Figura 3 que é derivada de uma série de 24 casos (com 28 sítios de hematomas). O acometimento de plano muscular profundo de parede póstero-lateral direita descrito neste relato de caso, é o quarto até agora descrito, se mostrando como um local de incomum localização dessa complicação da cirrose. A mortalidade associada à essa doença varia bastante, mas segundo as poucas séries de casos que foram encontradas feitas por: Sugiyama et al. ${ }^{1}$ e Takamura et al. ${ }^{3}$ ela pode chegar até $75 \%$ e $70,4 \%$, respectivamente o que não pode ser dito com certeza, mas indica para uma alta taxa de mortalidade dessa complicação nesse tipo específico de paciente.

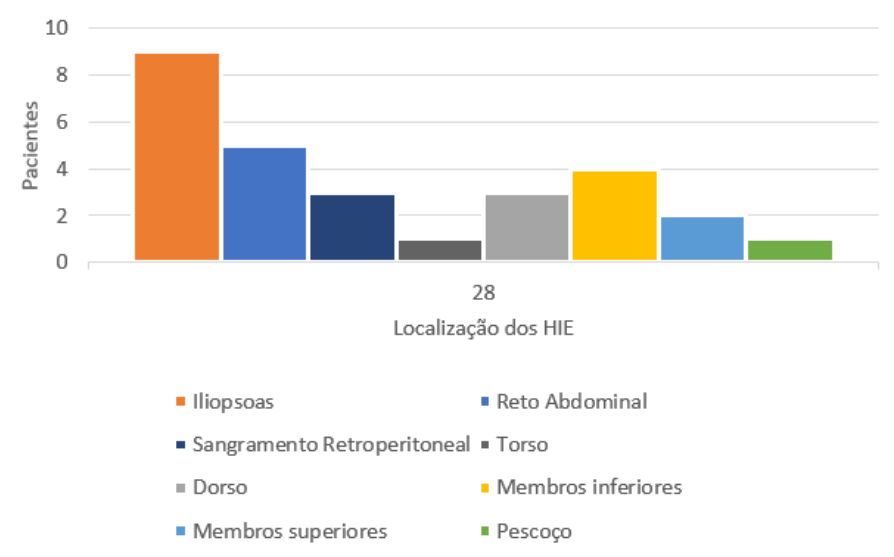

Figura 3: Localizações mais comuns de hematomas intramusculares espontâneos.

Não há consenso também entre os autores sobre qual a melhor forma de abordagem terapêutica em um paciente cirrótico com HIE dado o desequilíbrio das forças pró e anticoagulantes, havendo opções conservadoras com hemotransfusões ${ }^{1}$ e/ou administração de vitamina $\mathrm{K}$ e outras mais 
agressivas como embolização arterial ${ }^{1,3}$, transplante de fígado ${ }^{1}$ e ressecção do hematoma ${ }^{3}$. Nenhuma das opções citadas possui consistência para ser considerada a melhor alternativa como pode ser observada na Figura 4 que resume as condutas utilizadas em uma série de casos onde as taxas de óbito são altas independente da conduta tomada. Existem estudos como o de Zissin et al. ${ }^{8}$ que indicam a realização de embolização arterial, por obterem parada de sangramento em 19 dos 26 casos, mas não se pode tirar como a melhor conduta dado que em Sugiyama et al. ${ }^{1}$ a mortalidade associada à esse procedimento foi de $88,8 \%$. No que tange o hemotórax espontâneos são suspeita quando há o líquido pleural com hematócrito maior ou igual a $50 \%$ do sangue periférico e ausência de sinais de trauma, sua ocorrência está mais associada com eventos vasculares intratorácicos ou intraabdominais, infarto pulmonar com infecção necrotizante dos pulmões ${ }^{9}$, neoplasias e uso de medicações anticoagulantes ${ }^{4}$; tais etiologias estavam ausentes no paciente deste relato o que tornam seu surgimento mais interessante e de difícil manejo, pois, não foi encontrado na literatura um caso semelhante o que gera um desafio à equipe médica que não tem um correlato para basear suas condutas. Adicionalmente, não foi possível fazer a toracocentese para aspirar o líquido pleural e confirmar o diagnóstico de hemotórax, uma vez que após reunião clínica o procedimento foi contraindicado dado o risco de descompensamento agudo do quadro hemorrágico que já era grave dado a presença do HIE e do hemotórax, sendo assim, o diagnóstico foi apenas por exames de imagem (TC e USG) e a conduta tomada foi a mesma para o hematoma: conservadora com medidas de suporte para hepatopatia crônica (hemotransfusões guidas pela $\mathrm{Hb}$, analgesia e vitamina $\mathrm{K}$ ), foi considerado também realizar transfusão de plasma fresco congelado, mas foi deixada de lado dado o risco de aumento da pressão portal associado ao seu uso ${ }^{10}$. Por fim, após um mês de internamento seguindo esse protocolo, o paciente recebeu alta com melhora dos valores hematimétricos e sintomatológica. Mesmo assim, não pode-se dizer que esta é a melhor conduta a ser tomada em um caso como esse, pois, como já foi supracitado há uma ausência gritante de dados de qualidade para embasar a conduta da equipe sendo necessária muita discussão e individualizar as medidas.

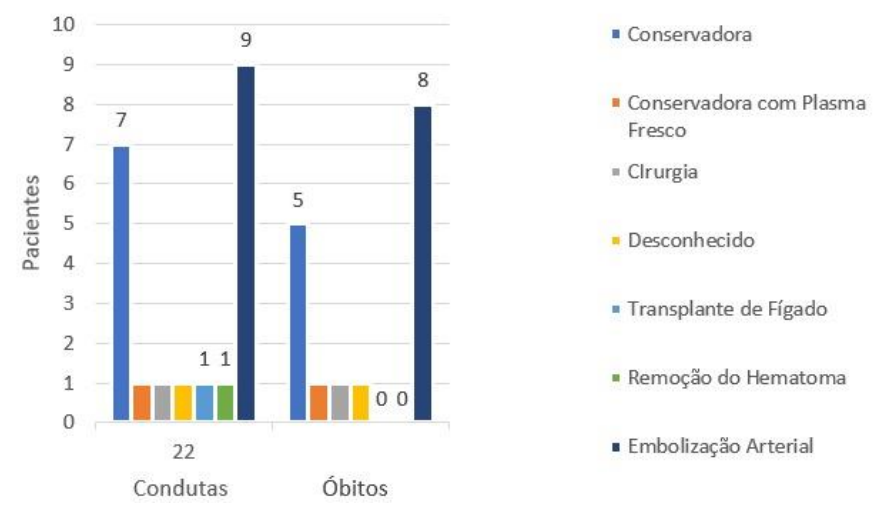

Figura 4: Levantamento de condutas realizadas em pacientes com HIE e mortalidade associada.

\section{Conclusão}

Como descrito anteriormente, o álcool é fator de risco para descompensação hepática e hemática, podendo ocasionar o surgimento do hematoma intramuscular. Conclui-se, dessa forma, que o manejo correto da situação faz com que condutas adequadas possam ser tomadas e 
diagnósticos precoces sejam obtidos, a fim de um melhor desfecho clínico dessa complicação da cirrose hepática. É importante salientar, ainda, que a individualidade de cada caso deve ser respeitada na decisão das condutas conservadoras ou agressivas, levando em consideração os desfechos clínicos particulares de cada paciente acometido. Por fim, considerando a frequência do hematoma intramuscular causado por doença hepática crônica de causa alcoólica e levando em conta sua alta morbimortalidade, faz-se necessária a notificação do caso para atualizações de literaturas.

\section{Referências}

1. Poordad FF (2015) Presentation and complications associated with cirrhosis of the liver. Curr Med Res Opin, 31(5):925-37.

2. Artigas MIO, Miguelena PRG (2015) Shock hipovolémico en paciente cirrótico secundario a hematoma del psoas. Gastroenterol Hepatol, 38(9):5667.

3. Takamura M, Watanabe J, Sakamaki A, Honda Y, Kamimura K, Tsuchiya A, et al. (2014) Alcoholic liver disease complicated by deep bleeding into the muscles or retroperitoneum: Report of three cases and a review of the literature. Intern Med, 53(16):1763-8.

4. Ali HA, Lippmann M, Mundathaje U, Khaleeq G (2008) Spontaneous hemothorax: A comprehensive review. Chest, 134(5):1056-65.

5. Sugiyama C, Akai A, Yamakita N, Ikeda T, Yasuda K (2009) Muscle hematoma: A critically important complication of alcoholic liver cirrhosis. World $\mathbf{J}$ Gastroenterol, 15(35):4457-60.

6. Craxì A, Cammà C, Giunta M (2000) Clinical aspects of bleeding complications in cirrhotic patients. Blood Coagul Fibrinolysis, 11(4 SUPPL. 1):75-80.

7. Cherry WB, Mueller PS (2006) Rectus sheath hematoma: Review of 126 cases at a single institution. Medicine (Baltimore), 85(2):105-10.

8. Zissin R, Gayer G, Kots E, Ellis M, Bartal G, Griton I (2007) Transcatheter arterial embolisation in anticoagulant-related haematoma - A current therapeutic option: A report of four patients and review of the literature. Int J Clin Pract, 61(8):1321-7.

9. Morgan CK, Bashoura L, Balachandran D, Faiz SA (2015) Spontaneous hemothorax. Ann Am Thorac Soc, 12(10):1578-82.

10. O'Leary JG, Greenberg CS, Patton HM, Caldwell SH (2019) AGA Clinical Practice Update: Coagulation in Cirrhosis. Gastroenterology, 157(1):34-43.

Autor para correspondência Maria Laryssa da Silva Pontes dralarypontes@gmail.com

Recebido: 30 de novembro de 2020 Aceito: 22 de dezembro de 2020 